\title{
A Note on Embedding the 4- arcs of Fano Plane with Quadric and Cubic Veroneseans to Projective Spaces
}

\author{
Ziya Akça* and Abdilkadir Altıntaş
}

\begin{abstract}
The 3 -spaces generate intersect pairwise in at least a line (each pair of 4 -sets share two points). But since the union of two such 4 -sets is a 6 -set, this union generates the whole space, so they pairwise intersect in a line. Dually, the lines that we consider have empty intersection. Other examples of such sets are spreads of lines, or spreads of any kind.

In this work, we present SCID properties spaces generated by 4 -arcs of Fano plane.
\end{abstract}

Keywords: Projective spaces; Fano plane; Arcs

AMS Subject Classification (2020): Primary: Ams Subject classification: 20 N25

${ }^{*}$ Corresponding author

\section{Introduction and Preliminaries}

Projective geometry provides a geometric way to study vector spaces. Indeed, a projective space over a skew field $K$ is nothing else than the lattice of proper non-trivial subspaces of a vector space over $K$. This is the origin of projective geometry.

The smallest example of a projective plane is the Fano projective plane over the field $G F(2)=\{0,1\}$. It is denoted by $P G(2,2)$. It is known that it has seven points and seven lines, and every line has exactly three points. Hence the Fano plane consists of the points $(1,0,0),(0,1,0),(0,0,1),(1,1,0),(1,0,1),(0,1,1)$ and $(1,1,1)$.

Let $P G(n, q)$ be the projective space of dimension $n$ over the Galois field $G F(q)$. A $k$-cap in $P G(n, q)$ is a set of $k$ points, no three of which are collinear. A $k$-cap in $P G(n, q)$ is called complete if it is not contained in a $(k+1)$-cap of $P G(n, q)$. If $n=2$, then a $k$-cap is usually called a $k$-arc.

The main questions on caps in $P G(n, q)$, which are also of interest in Coding Theory, concern the size of very large caps, especially near $m_{2}(n, q)$, the size of the largest complete cap, and near $m_{2}^{\prime}(n, q)$, the size of the second largest complete cap.

Veronesean varieties have a long and rich history, and were originally studied as classical real or complex varieties. But they can be defined over arbitrary fields. Over finite fields they have proved to be very useful tools in finite geometry. Given the importance of these objects in classical algebraic geometry and finite geometry, it is a wortwhile job to do. The simplest Veronesean varieties are the quadric Veronesans $\mathcal{V}_{n}$ of index $n$. In order to use their properties as tools in proofs, one has to recognize these varieties and so characterization theorems are very important [6]-[8]. In the literature one can find four different kinds of characterizations of the finite quadric Veroneseans. The Veronesean map defined from projective plane $P G(2, q)$ to projective space $P G(5, q)$ maps the set of points of each line to a set of coplanar points such that image of this map generates the projective space. The quadric Veronesean $\mathcal{V}_{2}$ has been studied in great detail, and characterized in different ways. Veronesean of $P G(n, q)$ will be denoted by $\mathcal{V}_{n}^{2^{n}}$ or simply $\mathcal{V}_{n}$. For $n=1$, the Veronesean $\mathcal{V}_{1}^{2}$ is a conic of $P G(2, q)$. For $n=2$, the Veronesean is a surface $\mathcal{V}_{2}^{4}$ of order 4 in $P G(5, q)$. For $n=3$, the Veronesean is a variety $\mathcal{V}_{3}^{8}$ of dimension 3 and order 8 of $P G(9, q)$. The classification of lax generalized Veronesean embeddings of projective spaces which relate strongly to the quadric Veronesean of these projective spaces have been studied in [1]-[4].

Received : 31-10-2019, Accepted : 23-10-2020 
Definition 1.1. The Veronese embedding of degree $d \in N$ is the map

$$
\begin{gathered}
V_{d}: \mathcal{P}^{n} \rightarrow \mathcal{P}^{\left(\begin{array}{c}
n+d \\
d
\end{array}\right)-1} \\
{\left[x_{0}, x_{1}, . ., x_{n}\right] \rightarrow\left[\left(x_{0}^{i_{0}}, . ., x_{n}^{i_{n}}\right)_{0 \leq i_{k} \leq \sum_{k=0}^{n} i_{k}=d}\right] .}
\end{gathered}
$$

Definition 1.2. Let $\mathcal{P}$ be a projective space, and let $-1 \leq k \lessdot n$. A set $E$ of $n$-dimensional subspaces of $\mathcal{P}$ with the property that any two elements of $E$ intersect precisely in a $k$-dimensional subspace is called an $(n ; k)-$ SCID (set of subspaces with constant intersection dimension).

Definition 1.3. Let $E$ be any SCID. Let $E_{1} ; E_{2} \in E$. Then each $k$-dimensional subspace of $E_{1} \cap E_{2}$ is called an intersection $k$-space of $E$. (for $k=0,1$ these are called intersection points, intersection lines, respectively).

For example, a (2,0)-SCID is a set of planes intersecting mutually in exactly one point.

Definition 1.4. An $t$-arc in a projective plane $\mathcal{P}$ is a collection of $t$ distinct points, no three of which are collinear.

These definitions have been taken from [7]-[8].

\section{Embedding The 4-Arcs of Fano Plane with Quadric Veronesean to PG $(5,2)$}

Veronesean embedding is an embedding

$$
\theta: P G(n, q) \rightarrow P G(n(n+3) / 2, q)
$$

such that $\theta$ maps the set of points of each line of $P G(n, q)$ to a set of coplanar points of $P G(n(n+3) / 2, q)$ and such that the image of $\theta$ generates $P G(n(n+3) / 2, q)$. $\theta$ is called Veronesean map $\mathcal{V}_{n}$ and the quadric Veronesean is the image of the Veronesean map. The quadric Veronesean $\mathcal{V}_{2}$ is one of the most important substructures in $P G(5, q)$. It is the image of the plane $P(2, q)$ under the mapping

$$
\begin{gathered}
\theta: P G(2, q) \rightarrow P G(5, q) \\
\left(x_{0}, x_{1}, x_{2}\right) \rightarrow\left(x_{0}^{2}, x_{1}^{2}, x_{2}^{2}, x_{0} x_{1}, x_{0} x_{2}, x_{1} x_{2}\right)
\end{gathered}
$$

Since the Fano plane consists of the points $(1,0,0),(0,1,0),(0,0,1),(1,1,0),(1,0,1),(0,1,1)$ and $(1,1,1)$.

The 4 -arcs of the projective plane $P G(2,2)$ are $\{(1,1,1),(1,1,0),(1,0,1),(1,0,0)\}$,

$\{(0,1,1),(1,1,0),(0,0,1),(1,0,0)\},\{(0,1,1),(1,1,1),(0,0,1),(1,0,1)\}$,

$\{(0,1,1),(1,1,1),(0,1,0),(1,1,0)\},\{(0,1,1),(0,1,0),(1,0,0),(1,0,1)\}$,

$\{(0,1,0),(1,1,0),(1,0,1),(0,0,1)\},\{(0,1,0),(1,0,0),(1,1,1),(0,0,1)\}$.

Under the quadric Veronesean mapping the points of 4 -arcs of Fano plane mapped to points in $P G(5,2)$ and these four points define a line in $P G(5,2)$. In fact the points of these lines are 3-dimensianoal subspaces of $P G(5,2)$.

Theorem 2.1. The quadric Veronesean of 4 -arcs of Fano plane form $(1,-1)-S C I D$ in $P G(5,2)$.

Proof. The quadric Veronesean mapping of 4 -arc $\{(1,1,1),(1,1,0),(1,0,1),(1,0,0)\}$ is

$$
\{(1,1,1,1,1,1),(1,1,0,1,0,0),(1,0,1,0,1,0),(1,0,0,0,0,0)\} .
$$

Let the points $(1,1,1,1,1,1),(1,1,0,1,0,0),(1,0,1,0,1,0),(1,0,0,0,0,0)$ generate a space $[a, b, c, d, e, f]$ in $P G(5,2)$. The equations $a=0, b=d, c=e, f=0$ are obtained. So these four points in $P G(5,2)$ form 3 -dimensional subspaces. These are $[0,0,1,0,1,0],[0,1,0,1,0,0],[0,1,1,1,1,0]$ in $P G(5,2)$. Since one of these 3 -spaces is linear combination of other two spaces, these spaces are regarded as points of a line. If this line is denoted by $L_{1}$, it consists of points $(0,0,1,0,1,0),(0,1,0,1,0,0),(0,1,1,1,1,0)$.

The quadric Veronesean mapping of another 4-arc $\{(0,1,1),(1,1,0),(0,0,1),(1,0,0)\}$ is

$$
\{(0,1,1,0,0,1),(1,1,0,1,0,0),(0,0,1,0,0,0),(1,0,0,0,0,0)\}
$$


. The points $(0,1,1,0,0,1),(1,1,0,1,0,0),(0,0,1,0,0,0),(1,0,0,0,0,0)$ generate a space $[a, b, c, d, e, f]$ in $P G(5,2)$. We get the equations $a=0, c=0, b=d=f$. So these four points in $P G(5,2)$ form 3-dimensional subspaces. We have three 3 -dimensional subspaces in the form $[0, b, 0, b, e, b]$. These are $[0,0,0,0,1,0],[0,1,0,1,1,1],[0,1,0,1,0,1]$ in $P G(5,2)$. Since one of these 3 -spaces is linear combination of other two spaces, these spaces are regarded as points of a line. If this line is denoted by $L_{2}$, it consists of the points $(0,0,0,0,1,0),(0,1,0,1,1,1),(0,1,0,1,0,1)$.

Similarly, if we compute the lines for other arcs, we have seven lines $L_{1}, L_{2}, \ldots L_{7}$ for seven 4 -arcs. These seven lines form $(1,-1)$-SCID in $P G(5,2)$ since any two of them have no intersection point.

\section{Embedding of 4-Arcs of Fano Plane with Cubic Veronesean to $\mathrm{PG}(9,2)$}

$$
\theta: \mathcal{P}^{2} \rightarrow \mathcal{P}^{3}
$$

such that $\theta$ maps $\left(x_{0}, x_{1}, x_{2}\right) \rightarrow\left(x_{0}^{3}, x_{1}^{3}, x_{2}^{3}, x_{0}^{2} x_{1}, x_{0}^{2} x_{2}, x_{1}^{2} x_{0}, x_{1}^{2} x_{2}, x_{2}^{2} x_{0}, x_{2}^{2} x_{1}, x_{0} x_{1} x_{2}\right)$.

Fano plane has seven 4 -arcs and under cubic Veronesean mapping the four points of 4-arc mapped to points in $P G(9,2)$ and these four points generate a 5 -space in $P G(9,2)$.

Theorem 3.1. The qubic Veronesean embedding of 4-arcs of Fano plane form $(5,3)-S C I D$ in $P G(9,2)$.

Proof. The cubic Veronesean mapping of 4 -arc $\{(0,1,0),(0,0,1),(1,0,0),(1,1,1)\}$ is

$$
\{(0,1,0,0,0,0,0,0,0,0),(0,0,1,0,0,0,0,0,0,0),(1,0,0,0,0,0,0,0,0,0),(1,1,1,1,1,1,1,1,1,1)\}
$$

Let the points $(0,1,0,0,0,0,0,0,0,0),(0,0,1,0,0,0,0,0,0,0),(1,0,0,0,0,0,0,0,0,0),(1,1,1,1,1,1,1,1,1,1)$ generate a space $[a, b, c, d, e, f, g, h, j, k]$ in $P G(9,2)$. We get the equations $a=0, b=0, c=0, d+e+f+g+h+j+k=0$. We have 63 subspaces in the form $a=0, b=0, c=0, d+e+f+g+h+j+k=0$. We can regard these 63 subpaces

\begin{tabular}{|c|c|c|}
\hline 1. $(0,0,0,0,0,0,0,0,1,1)$ & $22 .(0,0,0,0,1,0,1,1,0,1)$ & \\
\hline 2. $(0,0,0,0,0,0,0,1,0,1)$ & 23. $(0,0,0,0,1,0,1,1,1,0)$ & \\
\hline 3. $(0,0,0,0,0,0,0,1,1,0)$ & 24. $(0,0,0,0,1,1,0,0,0,0)$ & \\
\hline & 25. $(0,0,0,0,1,1,0,0,1,1)$ & $1,1,0,0)$ \\
\hline & & 4 \\
\hline & 27. $(0,0,0,0,1,1,0,1,1,0)$ & \\
\hline & & \\
\hline & & \\
\hline & & \\
\hline & & \\
\hline & & \\
\hline & & \\
\hline & & \\
\hline & & \\
\hline & & $, 0,1,0)$ \\
\hline $1,0,0,0,0,1)$ & $, 1,0,1,1)$ & $0,0)$ \\
\hline & & \\
\hline & & \\
\hline & & \\
\hline 20. $(0,0,0,0,1,0,1,0,0,0)$ & $0,0,1,0,1,0,0,1,1)$ & 62. \\
\hline 21. $(0,0,0,0,1,0,1,0,1,1)$ & 42. $(0,0,0,1,0,1,0,1,0,1)$ & 63. $(0,0,0,1,1,1,1,1,1,0)$ \\
\hline
\end{tabular}
as points of a 5 -space in $P G(9,2)$. If this 5 -space is denoted by $S_{1}$, its points are as the following:

The cubic Veronesean mapping of 4 -arc $\{(0,1,0),(0,1,1),(1,0,0),(1,0,1)\}$ is

$$
\{(0,1,0,0,0,0,0,0,0,0),(0,1,1,0,0,0,1,0,1,0),(1,0,0,0,0,0,0,0,0,0),(1,0,1,0,1,0,0,1,0,0)\}
$$

Let the points $(0,1,0,0,0,0,0,0,0,0),(0,1,1,0,0,0,1,0,1,0),(1,0,0,0,0,0,0,0,0,0),(1,0,1,0,1,0,0,1,0,0)$ form a space $[a, b, c, d, e, f, g, h, j, k]$ in $P G(9,2)$. The equations $a=0, b=0, h=c+e, j=c+g$ are obtained. We have 63 
subspaces in the form $a=0, b=0, e+g+h+j=0$. We can regard these 63 subpaces as points of a 5 -space in $P G(9,2)$. If this 5 -space is denoted by $S_{2}$, its points are as the following::

$$
\begin{aligned}
& \text { 1. }(0,0,0,0,0,0,1,0,1,0) \quad 22 .(0,0,0,0,1,1,1,1,1,0) \\
& \text { 2. }(0,0,0,0,0,1,0,0,0,0) \quad \text { 23. }(0,0,0,1,0,1,1,0,1,0) \\
& \text { 3. }(0,0,0,0,1,0,0,1,0,0) \quad \text { 24. }(0,0,0,1,1,0,1,1,1,0) \\
& \text { 4. }(0,0,0,1,0,0,0,0,0,0) \quad \text { 25. }(0,0,0,1,1,1,0,1,0,0) \\
& \text { 5. }(0,0,1,0,0,0,0,1,1,0) \quad \text { 26. }(0,0,1,1,1,1,0,0,1,0) \\
& \text { 6. }(0,0,0,0,0,1,1,0,1,0) \quad \text { 27. }(0,0,1,1,1,0,1,0,0,0) \\
& \text { 7. }(0,0,0,0,1,0,1,1,1,0) \quad \text { 28. }(0,0,1,1,0,1,1,1,0,0) \\
& \text { 8. }(0,0,0,0,1,1,0,1,0,0) \quad \text { 29. }(0,0,1,0,1,1,1,0,0,0) \\
& \text { 9. }(0,0,0,1,0,0,1,0,1,0) \quad 30 .(0,0,0,1,1,1,1,1,1,0) \\
& \text { 10. }(0,0,0,1,1,0,0,1,0,0) \quad 31 .(0,0,1,1,1,1,1,0,0,0) \\
& \text { 11. }(0,0,0,1,0,1,0,0,0,0) \quad 32 .(0,0,0,0,0,0,0,0,0,1) \\
& \text { 12. }(0,0,1,1,0,0,0,1,1,0) \quad 33 .(0,0,0,0,0,0,1,0,1,1) \\
& \text { 13. }(0,0,1,0,1,0,0,0,1,0) \quad 34 .(0,0,0,0,0,1,0,0,0,1) \\
& \text { 14. }(0,0,1,0,0,1,0,1,1,0) \quad 35 .(0,0,0,0,1,0,0,1,0,1) \\
& \text { 15. }(0,0,1,0,0,0,1,1,0,0) \quad 36 .(0,0,0,1,0,0,0,0,0,1) \\
& \text { 16. }(0,0,1,1,1,0,0,0,1,0) \quad 37 .(0,0,1,0,0,0,0,1,1,1) \\
& \text { 17. }(0,0,1,1,0,1,0,1,1,0) \quad 38 .(0,0,0,0,0,1,1,0,1,1) \\
& \text { 18. }(0,0,1,1,0,0,1,1,0,0) \quad 39 .(0,0,0,0,1,0,1,1,1,1) \\
& \text { 19. }(0,0,1,0,1,1,0,0,1,0) \quad \text { 40. }(0,0,0,0,1,1,0,1,0,1) \\
& \text { 20. }(0,0,1,0,0,1,1,1,0,0) \quad 41 .(0,0,0,1,0,0,1,0,1,1) \\
& \text { 21. }(0,0,1,0,1,0,1,0,0,0) \\
& \text { 42. }(0,0,0,1,1,0,0,1,0,1) \\
& \text { 43. }(0,0,0,1,0,1,0,0,0,1) \\
& \text { 44. }(0,0,1,1,0,0,0,1,1,1) \\
& \text { 45. }(0,0,1,0,1,0,0,0,1,1) \\
& \text { 46. }(0,0,1,0,0,1,0,1,1,1) \\
& \text { 47. }(0,0,1,0,0,0,1,1,0,1) \\
& \text { 48. }(0,0,1,1,1,0,0,0,1,1) \\
& \text { 49. }(0,0,1,1,0,1,0,1,1,1) \\
& \text { 50. }(0,0,1,1,0,0,1,1,0,1) \\
& \text { 51. }(0,0,1,0,1,1,0,0,1,1) \\
& \text { 52. }(0,0,1,0,0,1,1,1,0,1) \\
& \text { 53. }(0,0,1,0,1,0,1,0,0,1) \\
& \text { 54. }(0,0,0,0,1,1,1,1,1,1) \\
& \text { 55. }(0,0,0,1,0,1,1,0,1,1) \\
& \text { 56. }(0,0,0,1,1,0,1,1,1,1) \\
& \text { 57. }(0,0,0,1,1,1,0,1,0,1) \\
& \text { 58. }(0,0,1,1,1,1,0,0,1,1) \\
& \text { 59. }(0,0,1,1,1,0,1,0,0,1) \\
& \text { 60. }(0,0,1,1,0,1,1,1,0,1) \\
& \text { 61. }(0,0,1,0,1,1,1,0,0,1) \\
& \text { 62. }(0,0,0,1,1,1,1,1,1,1) \\
& \text { 63. }(0,0,1,1,1,1,1,0,0,1)
\end{aligned}
$$

One can easly see that the intersection of 5 -spaces $S_{1}$ and $S_{2}$ is a 3 -space in $P G(9,2)$, as follows

$$
S_{1} \cap S_{2}=\left\{\begin{array}{ll}
1 .(0,0,0,0,0,0,1,0,1,0), & 9 .(0,0,0,1,0,0,1,0,1,1), \\
2 .(0,0,0,0,0,1,0,0,0,1), & 10 .(0,0,0,1,0,1,0,0,0,0), \\
3 .(0,0,0,0,0,1,1,0,1,1), & 11 .(0,0,0,1,0,1,1,0,1,0), \\
4 .(0,0,0,0,1,0,0,1,0,0), & 12 .(0,0,0,1,1,0,0,1,0,1), \\
5 .(0,0,0,0,1,0,1,1,1,0), & 13 .(0,0,0,1,1,0,1,1,1,1), \\
6 .(0,0,0,0,1,1,0,1,0,1), & 14 .(0,0,0,1,1,1,0,1,0,0), \\
7 .(0,0,0,0,1,1,1,1,1,1), & 15 .(0,0,0,1,1,1,1,1,1,0), \\
8 .(0,0,0,1,0,0,0,0,0,1), &
\end{array}\right\}
$$

Similarly, if the spaces for other arcs are computed, seven 5 -spaces $S_{1}, S_{2}, \ldots S_{7}$ are obtained for seven 4 -arcs. Since any two of them intersetc on a 3 -space in $P G(9,2)$. These seven spaces form $(5,3)$-SCID in $P G(9,2)$.

Theorem 3.2. Let $S_{i}$ such that the image of $\theta, i=1,2, \ldots, 7$ be $(5,3)-S C I D$ in $P G(9,2)$. The set of the intersection of $(5,3)-S C I D S_{i}, i=1,2, \ldots, 7$ is a projective plane.

Proof. The set of the intersection of seven 5 -spaces $S_{1}, S_{2}, \ldots S_{7}$ in $P G(9,2)$ is consist of 7 points. That is,

$$
S_{1} \cap S_{2} \cap \ldots \cap S_{7}=\left\{\begin{array}{c}
N_{1}(0,0,0,0,1,0,0,1,0,0), N_{2}(0,0,0,1,0,1,0,0,0), N_{3}(0,0,0,0,0,0,1,0,1,0), \\
N_{4}(0,0,0,1,1,1,0,1,0,0), N_{5}(0,0,0,1,0,1,1,0,1,0), N_{6}(0,0,0,0,1,0,1,1,1,0) \\
N_{7}(0,0,0,1,1,1,1,1,1,0) .
\end{array}\right\}
$$

It can be seen this set of points forms a projective plane.

\section{Acknowledgments}

This work was supported by the Scientific Research Projects Commission of Eskisehir Osmangazi University under Project Number 2019-2542.

\section{References}

[1] Akça, Z., Bayar, A., Ekmekçi, S., Kaya, R., Thas, J. A., Van Maldeghem, H.: Generalized Veronesean embeddings of projective spaces, Part II. The lax case, Ars Combinatoria, Volume CIII, January, 2012, 65-80. 
[2] Akça, Z., Bayar, A., Ekmekçi, S., Kaya, R., Van Maldeghem, H.: Bazı Geometrilerin Sonlu Projektif Uzaylara Gömülmeleri Üzerine, Tübitak Proje No: 108T340, 2010

[3] Bayar, A., Akça, Z., Ekmekçi, S.: 4.Mertebeden Projektif Düzlemin 4-boyutlu Projektif Uzaya Gömülmesi Üzerine, Ogü Bap Proje No: 201619D38, 2017.

[4] Ekmekçi, S., Bayar, A., Akça, Z.: PG(4,4) Projektif Uzayındaki Projektif Düzlemler Üzerine, Ogü Bap Proje No: 201619D37, 2017.

[5] Hirschfeld, H., Thas.JJ. A.: 2016, General Galois Geometries, Springer Monongraphs in Mathematics.

[6] Thas, J.A., Van Maldeghem, H.: Characterizations of quadric and Hermitian Veroneseans over finite fields, J. of Geometry, 76, 282-293 (2003).

[7] Thas, J. A., Van Maldeghem, H.: Classification of finite Veronesean caps, European J. of Combin. 25, 275-285 (2004).

[8] Thas, J. A., Van Maldeghem, H.: Characterizations of the finite quadric Veroneseans, Quart. J. Math, 55, 99-113 (2004).

\section{Affiliations}

ZIYA AKÇA

AdDRESS: Eskişehir Osmangazi University, Faculty of Science and Letters, Department of Mathematics and Computer Science, 26480 Eskişehir, Turkey.

E-MAIL: zakca@ogu.edu.tr

ORCID ID: 0000-0001-6379-0546

ABDilKadir Altintaş

AdDress: Eskişehir Osmangazi University, Faculty of Science and Letters, Department of Mathematics and Computer Science, 26480 Eskişehir, Turkey.

E-MAIL: kadiraltintas1977@gmail.com

ORCID ID: 0000-0002-7012-352X 